\title{
TRANSITIONS BETWEEN NONSYMMETRIC AND SYMMETRIC STEADY STATES NEAR A TRIPLE EIGENVALUE*
}

\author{
D. S. COHEN $\dagger$ AND T. ERNEUX $† \ddagger$
}

\begin{abstract}
We examine the existence of nonuniform steady-state solutions of a certain class of reactiondiffusion equations. Our analysis concentrates on the case where the first bifurcation is near a triple eigenvalue. We derive the conditions for a continuous transition between nonsymmetric and symmetric solutions when the bifurcation parameter progressively increases from zero. Finally, we give an example of a four variables model which presents the possibility of a triple eigenvalue.
\end{abstract}

1. Introduction. Numerous experiments on growing and regenerating systems reveal that cellular differentiation is essentially a two step process. In the first step, it is assumed that a concentration gradient of a substance, or a gradient of some other physical variable, is formed in a cell population. The level of this gradient in any cell assigns a specific state to each unit in this population. In the second step, the cells differentiate according to their position and their genetic program [17], [18].

In order to explain the emergence of concentration patterns in a previously homogeneous medium, several authors have proposed that these chemical structures appear as the result of the interactions of reactive and diffusive substances [6], [7], [13], [14]. Much of the theoretical work of chemical networks involving diffusion and reaction has been devoted to the following set of nonlinear partial differential equations.

$$
\begin{aligned}
& \frac{\partial}{\partial t} X=F(X, \lambda, \gamma, \mu)+D \frac{\partial^{2}}{\partial r^{2}} X, \quad 0 \leqq r \leqq l, \\
& \frac{\partial}{\partial r} X=0, \quad r=0, l, \quad X(0, r)=X_{i}(r),
\end{aligned}
$$

where $X=\operatorname{col}\left(X_{1}, X_{2}, \cdots X_{n}\right), F(X, \lambda, \gamma, \mu)$ represents the reaction kinetics associated to the intermediates $X_{i}$ and $D$ is the matrix of constant diffusion coefficients. $\lambda$, $\gamma, \mu$ correspond to physico-chemical control parameters.

Recently, we have studied the possible coexistence of steady-state solutions of equations (1.1) which present 1 and 2 as basic wave numbers and near a double eigenvalue. We have shown that a continuous evolution between these structures is possible even if parameters are nonuniformly distributed in the system [2] (this issue, pp. 1042-1060). The predictions of our analysis were compared to experiments involving transitions between polar (nonsymmetric) and symmetric patterns [3]. Since different transformations are sometimes observed when the normal development is perturbed, we shall examine a different possibility which may influence the transition between nonsymmetric and symmetric steady states.

The purpose of this paper is to analyze the existence of steady state solutions near a triple degenerate bifurcation point. Although, the bifurcation diagram of the steady states is more complex, our analysis, given in $\S 2$, indicates that a direct transition between steady state solutions presenting 1 and 2 as basic wave numbers can still be expected. Since our study is based on the assumption of the existence of triple degenerate bifurcation points, we give in $\S 3$ an example of a model system presenting this possibility. Section 4 discusses the results.

\footnotetext{
* Received by the editors January 7, 1981, and in revised form October 20, 1982.

† Department of Applied Mathematics, California Institute of Technology, Pasadena, California 91125.

$\ddagger$ Present address: Department of Engineering Sciences and Applied Mathematics, Northwestern University, Evanston, Illinois 60201.
} 
2. Bifurcation near a triple eigenvalue. In this section, we analyze the steady state bifurcation from the trivial solution of the reaction-diffusion equations (1.1). The present analysis concentrates on the case when the first bifurcation is from a triple eigenvalue.

Let us introduce a steady state solution $X=X_{0}$ which obeys the equation

$$
F\left(X_{0}, \lambda, \gamma, \mu\right)=0 \text {. }
$$

It is convenient to define the new variables

$$
\begin{gathered}
x \equiv X-X_{0}, \\
s \equiv \frac{r}{l}
\end{gathered}
$$

and rewrite (1.1) in terms of $x$ and $s$ :

$$
\begin{aligned}
& \frac{\partial}{\partial t} x=L(\lambda, \gamma, \mu) x+H(x, \lambda, \gamma, \mu), \quad 0 \leqq s \leqq 1, \\
& \frac{\partial}{\partial s} x=0, \quad s=0,1, \quad x(0, s)=x_{i}(s)
\end{aligned}
$$

where we have fixed the values of all the parameters except $\lambda, \gamma$ and $\mu . L(\lambda, \gamma, \mu)$ is defined as $L(\lambda, \gamma, \mu)=\left(\partial F / \partial X_{j}\right)_{X_{0}}+\left(D / l^{2}\right) \partial^{2} / \partial s^{2}$, and $H(x, \lambda, \gamma, \mu)$ corresponds to the nonlinear part of $F(X, \lambda, \gamma, \mu)$ after introducing (2.2a).

We assume that (2.3) satisfies the following conditions:

1. We assume that when $\lambda$ progressively increases from zero, there exists a domain of $(\lambda, \gamma, \mu)$ values where $x=0$ is stable to perturbations uniform in space and the first bifurcation point of the basic state corresponds to nonuniform steady states. To determine the eventual branching of steady, nonuniform solutions of equation (2.3), it is necessary to solve the linear eigenvalue problem

$$
L(\lambda, \gamma, \mu) u=0 .
$$

We assume that (2.4) admits nontrivial solutions when

$$
\lambda=\lambda_{n}(\gamma, \mu), \quad n=1,2, \cdots \text {. }
$$

The corresponding eigenfunctions take the simple form

$$
u_{n}=p_{n} \cos n \pi s,
$$

where $p_{n}$ is a constant vector, satisfying

$$
\left\{\left(\frac{\partial F}{\partial X_{j}}\right)_{X_{0}, \lambda_{n}}-\frac{D}{l^{2}} k_{n}^{2}\right\} p_{n}=0, \quad k_{n}^{2}=n^{2} \pi^{2} .
$$

2. There exist critical values of $\gamma=\gamma^{0}, \mu=\mu^{0}$ such that

$$
\lambda=\lambda_{1}\left(\gamma^{0}, \mu^{0}\right)=\lambda_{2}\left(\gamma^{0}, \mu^{0}\right)=\lambda_{3}\left(\gamma^{0}, \mu^{0}\right) .
$$

Moreover, when $\lambda$ increases from zero, we assume that $\lambda=\lambda^{0}$ corresponds to the first bifurcation point of $x=0$. Note that in contrast to the general case $\left(\gamma \neq \gamma^{0}, \mu \neq \mu^{0}\right)$ the three eigenfunctions $u_{n}(n=1,2,3)$ given by (2.6) are all in the nullspace $N$ of $L_{0} \equiv L\left(\lambda^{0}, \gamma^{0}, \mu^{0}\right)$ :

$$
L_{0} u_{n}^{0}=0, \quad n=1,2,3 .
$$


An example of a model chemical system operating in one space dimension and satisfying these two hypotheses is presented in $\S 3$. Triple degenerate bifurcation points can also be observed for symmetric two- or three-dimensional systems. An example has been studied in the context of convective instabilities [4] and another reaction-diffusion problem has been examined by Reiss [15].

In the sequel, we intend to solve equations $(2.3)$ with $\partial x / \partial t=0$ for $(\lambda, \gamma, \mu)$ near $\left(\lambda^{0}, \gamma^{0}, \mu^{0}\right)$ using a generalization of the method proposed by Bauer et al. [1], [2], [5], [8], [9], [11], [16]. The bifurcation analysis will involve three parameters: we define $\delta=\lambda-\lambda^{0}, \sigma=\gamma-\gamma^{0}, \omega=\mu-\mu^{0}$ and rewrite (2.3) with $\partial x / \partial t=0$ in terms of $\delta, \sigma, \omega$ :

$$
\begin{aligned}
& M(\delta, \sigma, \omega) x+N(x, \delta, \sigma, \omega)=0, \quad 0 \leqq s \leqq 1, \\
& \frac{d x}{d s}=0, \quad s=0,1,
\end{aligned}
$$

where

$$
\begin{aligned}
& M(\delta, \sigma, \omega)=L\left(\lambda^{0}+\delta, \gamma^{0}+\sigma, \mu^{0}+\omega\right), \\
& N(x, \delta, \sigma, \omega)=H\left(x, \lambda^{0}+\delta, \gamma^{0}+\sigma, \mu^{0}+\omega\right) .
\end{aligned}
$$

To solve (2.10) for $\delta, \sigma, \omega$ small, we first define $\varepsilon$ as

$$
\varepsilon=\lambda_{2}-\lambda_{1} \quad(0<\varepsilon \ll 1)
$$

and assume the following expansions for $\lambda_{3}-\lambda_{1}$ and the bifurcation parameter $\delta$ :

$$
\begin{aligned}
& \lambda_{3}-\lambda_{1}=\varepsilon a_{1}+\varepsilon^{2} a_{2} \cdots>0, \\
& \delta=\varepsilon \delta_{1}+\varepsilon^{2} \delta_{2}+\cdots
\end{aligned}
$$

Then, we seek a solution of (2.10) of the form,

$$
x=x(s, \varepsilon)=\varepsilon x_{1}(s)+\varepsilon^{2} x_{2}(s)+\cdots .
$$

From (2.12), (2.13) and since $\lambda_{j}=\lambda_{j}(\sigma, \omega)$ we find that

$$
\begin{aligned}
& \sigma=\varepsilon \sigma_{1}+\varepsilon^{2} \sigma_{2}+\cdots, \\
& \omega=\varepsilon \omega_{1}+\varepsilon^{2} \omega_{2}+\cdots .
\end{aligned}
$$

Moreover, in order to carry out our perturbation procedure, we assume that $M(\delta, \sigma, \omega)$ and $Q(x, \delta, \sigma, \omega)$ have the following representations:

$$
M(\delta, \sigma, \omega)=L_{0}+\delta M_{1}+\sigma M_{2}+\omega M_{3}+O\left(\delta^{2}, \sigma^{2}, \omega^{2}, \delta \sigma, \delta \omega, \sigma \omega\right)
$$

when $|\delta|=O(\sigma)=O(\omega) \rightarrow 0$,

$$
Q(x, \delta, \sigma, \omega)=Q_{2}(x, x, \delta, \sigma, \omega)+Q_{3}(x, x, x, \delta, \sigma, \omega)+\cdots
$$

when $|x| \rightarrow 0 . Q_{k}(x, \cdots, x, \delta, \sigma, \omega)$ represents vectors of homogeneous polynomials of degree $k$ in the concentration variables $x_{1}, \cdots, x_{n}$. From (2.12)-(2.14) and the definition of $\delta$, we find that

$$
\lambda-\lambda_{j}=\varepsilon P_{j}+\varepsilon^{2} Q_{j}+\cdots \quad(j=1,2,3),
$$

where the coefficients $P_{j}, Q_{j} \cdots$ are linear combinations of $\delta_{1}, \delta_{2}, \sigma_{1}, \sigma_{2}, \cdots, \omega_{1}$, $\omega_{2}, \cdots$. Moreover, from (2.12), (2.13) and (2.20), we obtain relations between the 
$P_{j}, Q_{j}, \cdots:$

$$
\begin{array}{ll}
P_{2}=P_{1}-1, & Q_{2}=Q_{1}, \\
P_{3}=P_{1}-a_{1}, & Q_{3}=Q_{1}-a_{2}, \cdots .
\end{array}
$$

Our bifurcation analysis will present three parts:

1. We determine the different unknown functions $x_{j}(s)$ appearing in the expansion (2.15).

2. We examine the question of secondary bifurcation.

3 . We develop an inner expansion of the solutions of $(2.10)$ when the general expansion (2.15) becomes no longer valid.

2.1. Bifurcation analysis. Introducing (2.14)-(2.17) into (2.10) and equating like powers of $\varepsilon$, we obtain the following equations for $x_{1}, x_{2}$ :

$$
\begin{aligned}
& L_{0} x_{1}=0, \quad 0 \leqq s \leqq 1, \\
& \frac{d x_{1}}{d s}=0, \quad s=0,1, \\
& L_{0} x_{2}=-\left(\delta_{1} M_{1}+\sigma_{1} M_{2}+\omega_{1} M_{3}\right) x_{1}-Q_{2}\left(x_{1}, x_{1}, 0,0,0\right), \quad 0 \leqq s \leqq 1, \\
& \frac{d x_{2}}{d s}=0, \quad s=0,1 .
\end{aligned}
$$

As a consequence of (2.9), the general solution of equations (2.22) is

$$
x_{1}(s)=\sum_{j=1}^{3} \alpha_{j} u_{j}^{0}
$$

where $\alpha_{1}, \alpha_{2}, \alpha_{3}$ are undetermined coefficients. Introducing (2.24) into the right-hand side of (2.23), we apply the solvability conditions

$$
\begin{aligned}
\int_{0}^{1} d s\left(\left[\left(\delta_{1} M_{1}\right.\right.\right. & \left.+\sigma_{1} M_{2}+\omega_{1} M_{3}\right) x_{1}(s) \\
& \left.\left.+Q_{2}\left(x_{1}(s), x_{1}(s), 0,0,0\right)\right], u_{j}^{*}\right)=0, \quad j=1,2,3,
\end{aligned}
$$

where $u_{j}^{*}(j=1,2,3)$ denote the three solutions which span the null space $N^{*}$ of the adjoint operator $L_{0}^{*}$ and satisfy, $\int_{0}^{1} d s\left(u_{j}^{0}, u_{j}^{*}\right)=1(j=1,2,3)$. These conditions are given by

$$
\begin{aligned}
& P_{1} \alpha_{1}+A_{1} \alpha_{1} \alpha_{2}+A_{2} \alpha_{2} \alpha_{3}=0, \\
& P_{2} \alpha_{2}+B_{1} \alpha_{1} \alpha_{3}+B_{2} \alpha_{1}^{2}=0, \\
& P_{3} \alpha_{3}+C_{1} \alpha_{1} \alpha_{2}=0,
\end{aligned}
$$

where $A_{1}, A_{2}, B_{1}, B_{2}, C_{1}$ are coefficients which are independent of $\delta, \sigma, \omega$ and are defined in Appendix A. The solutions of (2.26) are given by:

(i)

(2.27) $\alpha_{1}=\alpha_{2}=\alpha_{3}=0$.

(ii)

(2.28) $\quad \alpha_{1}=\alpha_{2}=0, \quad P_{3}=0, \quad \alpha_{3}$ undetermined.

(iii)

$$
\alpha_{1}=\alpha_{3}=0, \quad P_{2}=0, \quad \alpha_{2} \text { undetermined } .
$$


(iv) When

$$
\Delta=\left(A_{1} P_{3}\right)^{2}+4 A_{2} C_{1} P_{1} P_{3} \geqq 0,
$$

$\alpha_{2}$ is given by

$$
\alpha_{2}=\frac{A_{1} P_{3} \pm \sqrt{\Delta}}{2 A_{2} C_{1}}
$$

and $\alpha_{1}, \alpha_{3}$ are related to $\alpha_{2}$ by

$$
\begin{gathered}
\alpha_{1}^{2}=\frac{P_{2} \alpha_{2}}{\left(B_{1} C_{1} \alpha_{2} / P_{3}-B_{2}\right)} \geqq 0, \\
\alpha_{3}=-\frac{C_{1} \alpha_{1} \alpha_{2}}{P_{3}} .
\end{gathered}
$$

Equation (2.27) corresponds to the basic state, (2.28), (2.29) describe vertical branches of solutions and (2.30) represents four distinct branches of solutions with $\alpha_{j} \neq 0$ $(j=1,2,3)$ provided the conditions $(2.30 \mathrm{a})$ and $(2.30 \mathrm{c})$ are satisfied. Two of these branches may present a singular behavior $\left(\left|\alpha_{1}\right| \rightarrow \infty\right)$ when

$$
\alpha_{2} \rightarrow \alpha_{2}^{*}=\frac{B_{2} P_{3}^{*}}{B_{1} C_{1}} .
$$

Introducing (2.31) into (2.30b) and using (2.21), we obtain the following conditions:

$$
\begin{aligned}
& P_{1}^{*}=\frac{a_{1} B_{2}\left(A_{1} B_{1}-A_{2} B_{2}\right)}{\left(A_{1} B_{1} B_{2}-A_{2} B_{2}^{2}+B_{1}^{2} C_{1}\right)}, \\
& \left(P_{1}^{*}-a_{1}\right)\left(A_{1}^{2}\left(P_{1}^{*}-a_{1}\right)+4 A_{2} C_{1} P_{1}^{*}\right) \geqq 0 .
\end{aligned}
$$

When $B_{2}<1$, (2.32), (2.33) become:

$$
P_{1}^{*} \simeq \frac{a_{1} B_{2} A_{1}}{B_{1} C_{1}}<1, \quad a_{1}^{2} A_{1}^{2}>0,
$$

and the singular point is located near $\lambda=\lambda_{1}+O\left(\varepsilon^{2}\right)$. When $B_{2}$ is larger, the amplitude $\alpha_{2}=\alpha_{2}\left(P_{1}\right)$ does not change- $(2.30 \mathrm{~b})$ is independent of $B_{2}$-but the value of $P_{1}^{*}$ increases. Figures 1, 2 illustrate the behavior of the bifurcation diagram when $B_{2}$ varies for particular values of the coefficients $A_{1}, A_{2}, B_{1}, B_{2}, C_{1}$ and $a_{1}$. In order to analyze the behavior of the solutions near the critical point defined by (2.32), (2.33), we need an inner expansion of the solutions of (2.10). This is presented in $\S 2.3$.

On the other hand, the next order corrections of the solutions (2.30) become singular when $P_{2} \rightarrow 0$ (or $P_{3} \rightarrow 0$ ). When $P_{2}$ (or $P_{3}$ ) approaches zero, we note from (2.30) that $\alpha_{2}=O(1), \alpha_{1}=O\left(\left|P_{2}\right|^{1 / 2}\right), \alpha_{3}=O\left(\left|P_{2}\right|^{1 / 2}\right)$, (or $\alpha_{3}=O(1), \alpha_{1}=O\left(\left|P_{3}\right|^{1 / 2}\right)$, $\left.\alpha_{2}=O\left(\left|P_{3}\right|^{1 / 2}\right)\right)$. These observations suggest that we reexamine the bifurcation problem when $P_{2}=0$ (or $P_{3}=0$ ) and seek a solution of (2.10) of the form

$$
x=X\left(s, \varepsilon^{1 / 2}\right)=\varepsilon^{1 / 2} X_{1}(s)+\varepsilon X_{2}(s)+\varepsilon^{3 / 2} X_{3}(s)+\cdots,
$$

assuming

$$
\lambda-\lambda_{2}=\varepsilon^{2} Q_{2}+O\left(\varepsilon^{3}\right) \quad\left(\text { or } \lambda-\lambda_{3}=\varepsilon^{2} Q_{3}+O\left(\varepsilon^{3}\right)\right) .
$$

In the next section, we examine each case and prove the existence of secondary bifurcations. 


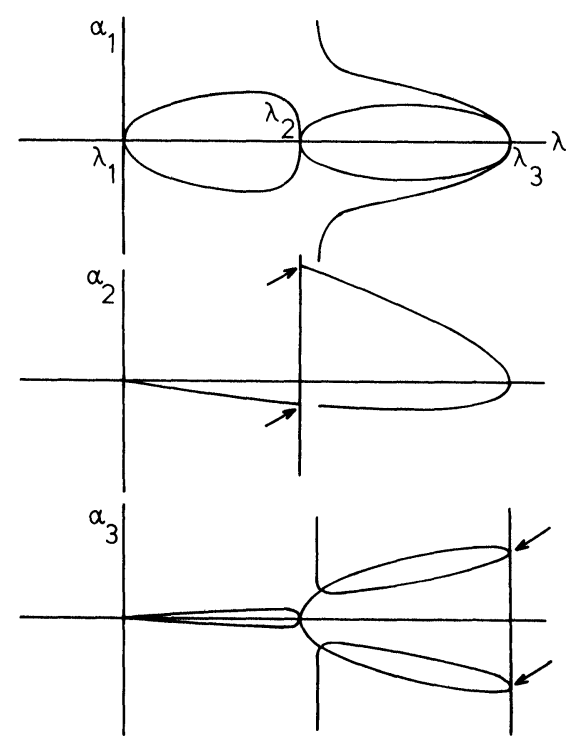

FIG. 1. Bifurcation diagram of the solutions of equations (2.26). We represent $\alpha_{1}, \alpha_{2}, \alpha_{3}$ as functions of the bifurcation parameter $\lambda$. The three amplitudes are given by (2.27)-(2.30). The values of the coefficients appearing in (2.26) are $A_{1}=2, A_{2}=-1, B_{1}=-2, B_{2}=-1.125, C_{1}=1.5$ and $a_{1}=2.2$. We observe secondary bifurcation points which are indicated by the arrows and a singular point where the branch of steady states becomes unbounded.

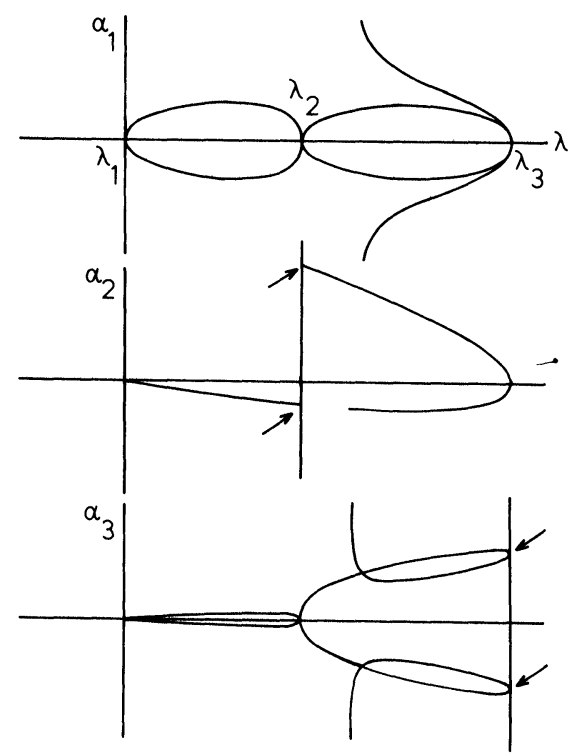

FIG. 2. Bifurcation diagram of the solutions of (2.26). As in Fig. 1, we represent the three amplitudes $\alpha_{1}, \alpha_{2}, \alpha_{3}$ as functions of $\lambda$. The values of the coefficients in (2.26) are the same as for Fig. 1 except $B_{2}=-1.5$.

\subsection{Secondary bifurcation.}

(i) $P_{2}=0$. We assume that the bifurcation parameter $\delta$ admits the following expansion:

$$
\delta=\varepsilon \delta_{1 c}+\varepsilon^{2} \delta_{2}+O\left(\varepsilon^{3}\right),
$$

where $\delta_{1}=\delta_{1 c}\left(\sigma_{1}, \omega_{1}\right)$ is fixed by the condition $P_{2}\left(\delta_{1 c}, \sigma_{1}, \omega_{1}\right)=0$. Consequently, from (2.20), (2.21) we observe the following relations between $\lambda$ and the three primary 
bifurcation points:

$$
\begin{gathered}
\lambda-\lambda_{2}=\varepsilon^{2} Q_{2}+O\left(\varepsilon^{3}\right), \\
\lambda-\lambda_{1}=\varepsilon+\varepsilon^{2} Q_{2}+O\left(\varepsilon^{3}\right), \\
\lambda-\lambda_{3}=\varepsilon\left(1-a_{1}\right)+\varepsilon^{2}\left(Q_{2}-a_{2}\right)+O\left(\varepsilon^{3}\right) .
\end{gathered}
$$

Introducing (2.16), (2.17), (2.35) and (2.37) into (2.10) and equating to zero the coefficients of each power of $\varepsilon^{1 / 2}$, we obtain a sequence of linear systems. Applying the solvability conditions, we find that

$$
x=\varepsilon\left(\beta_{2}+O\left(\varepsilon^{1 / 2}\right)\right) u_{2}^{0}+\varepsilon^{3 / 2}\left(\beta_{1} u_{1}^{0}+\beta_{3} u_{3}^{0}\right)+O\left(\varepsilon^{2}\right),
$$

where $u_{j}^{0}(j=1,2,3)$ are defined by (2.9) and $\beta_{j}(j=1,2,3)$ satisfy,

$$
\begin{aligned}
& \beta_{1}+A_{1} \beta_{1} \beta_{2}+A_{2} \beta_{3} \beta_{2}=0, \\
& Q_{2} \beta_{2}+B_{1} \beta_{1} \beta_{3}+B_{2} \beta_{1}^{2}+B_{3} \beta_{2}^{3}=0, \\
& \left(1-a_{1}\right) \beta_{3}+C_{1} \beta_{1} \beta_{2}=0
\end{aligned}
$$

and the new coefficient $B_{3}$ is defined in Appendix B. Equations (2.40) admit the following solutions:

(i)

$$
\beta_{1}=\beta_{2}=\beta_{3}=0
$$

(ii) If $Q_{2} / B_{3}<0$

$$
\beta_{1}=\beta_{3}=0, \quad \beta_{2}= \pm\left(-Q_{2} / B_{3}\right)^{1 / 2}
$$

(iii) When

$$
\Delta=A_{1}^{2}\left(1-a_{1}\right)^{2}+4 A_{2} C_{1}\left(1-a_{1}\right) \geqq 0,
$$

$\beta_{2}$ is a constant given by

$$
\beta_{2}^{0}=\frac{A_{1}\left(1-a_{1}\right) \pm \sqrt{\Delta}}{2 A_{2} C_{1}}
$$

and $\beta_{1}, \beta_{3}$ are functions of $Q_{2}$ :

$$
\begin{gathered}
\beta_{1}^{2}=\frac{\beta_{2}^{0}\left(Q_{2}+B_{3} \beta_{2}^{0^{2}}\right)}{\left(B_{1} C_{1} \beta_{2}^{0} /\left(1-a_{1}\right)-B_{2}\right)} \geqq 0, \\
\beta_{3}=-\beta_{1} \beta_{2}^{0} C_{1} /\left(1-a_{1}\right) .
\end{gathered}
$$

Equation (2.41) corresponds to the basic state, (2.42) represents primary bifurcating states appearing at $Q_{2}=0\left(\lambda=\lambda_{2}+O\left(\varepsilon^{3}\right)\right),(2.43)$ describes secondary bifurcating steady states emerging from (2.42) at $Q_{2}=-B_{3} \beta_{2}^{0^{2}}$ and connecting when $\left|Q_{2}\right| \rightarrow \infty$ the primary steady state solutions previously described in $\S 2$ when $P_{2} \neq 0$. Note that if condition (2.43a) is verified, condition (2.43c) is always satisfied since $Q_{2}+B_{3} \beta_{2}^{0^{2}}$ can be positive or negative.

(ii) $P_{3}=0$. We assume an expansion of $\delta$ of the form (2.37) where $\delta_{1 c}\left(\sigma_{1}, \omega_{1}\right)$ is fixed by the condition $P_{3}\left(\delta_{1 c}, \sigma_{1}, \omega_{1}\right)=0$. From (2.20), (2.21), we observe the following relations between $\lambda$ and the primary bifurcation points:

$$
\begin{aligned}
& \lambda-\lambda_{3}=\varepsilon^{2} Q_{3}+O\left(\varepsilon^{3}\right), \\
& \lambda-\lambda_{1}=\varepsilon a_{1}+\varepsilon^{2}\left(Q_{3}+a_{2}\right)+O\left(\varepsilon^{3}\right),
\end{aligned}
$$


$(2.44 c)$

$$
\lambda-\lambda_{2}=\varepsilon\left(a_{1}-1\right)+\varepsilon^{2}\left(Q_{3}+a_{2}\right)+O\left(\varepsilon^{3}\right) .
$$

Introducing (2.16), (2.17), (2.35) and (2.37) into (2.10), equating to zero the coefficients of each power of $\varepsilon^{1 / 2}$ and applying the solvability conditions, we find the following results:

$$
x=\varepsilon\left(\beta_{3}+O\left(\varepsilon^{1 / 2}\right)\right) u_{3}^{0}+\varepsilon^{3 / 2}\left(\beta_{1} u_{1}^{0}+\beta_{2} u_{2}^{0}\right)+O\left(\varepsilon^{2}\right),
$$

where the amplitudes $\beta_{1}, \beta_{2}, \beta_{3}$ satisfy

$$
\begin{aligned}
& a_{1} \beta_{1}+A_{2} \beta_{2} \beta_{3}=0, \\
& \left(a_{1}-1\right) \beta_{2}+B_{1} \beta_{1} \beta_{3}=0, \\
& Q_{3} \beta_{3}+C_{1} \beta_{1} \beta_{2}+C_{2} \beta_{3}^{3}=0,
\end{aligned}
$$

where $C_{2}$ is defined in Appendix C. Equations (2.46) admit the following solutions: (i)

$$
\beta_{1}=\beta_{2}=\beta_{3}=0 .
$$

(ii) If $Q_{3} / C_{2}<0$,

$$
\beta_{1}=\beta_{2}=0, \quad \beta_{3}= \pm\left(-Q_{3} / C_{2}\right)^{1 / 2} .
$$

(iii) $\beta_{3}$ is a constant given by

$$
\beta_{3}^{02}=a_{1}\left(a_{1}-1\right) / A_{2} B_{1}>0
$$

and $\beta_{1}, \beta_{2}$ depend on $Q_{3}$ :

$$
\begin{aligned}
& \beta_{1}^{2}=\left(a_{1}-1\right)\left(Q_{3}+C_{2} \beta_{3}^{02}\right) / B_{1} C_{1}>0, \\
& \beta_{2}^{2}=a_{1}\left(Q_{3}+C_{2} \beta_{3}^{02}\right) / A_{2} C_{1}>0 .
\end{aligned}
$$

Equation (2.47) is the basic state, (2.48) represents primary bifurcating states emerging from $x=0$ at $Q_{3}=0\left(\lambda=\lambda_{3}+O\left(\varepsilon^{3}\right)\right)$, (2.49) describes secondary bifurcating states emerging from (2.48) at $Q_{3}=-C_{2} \beta_{3}^{02}$ provided the three conditions (2.49a)-(2.49c) can be satisfied. Moreover, when $\left|Q_{3}\right| \rightarrow \infty$, they connect the primary branches of solutions described in $\S(2.1)$ when $P_{3} \neq 0$.

2.3. Inner expansion of the steady state solutions near the singular point $P_{1}=$ $\boldsymbol{P}_{\mathbf{1}}^{*}$. When the general expansion of the steady state solutions presents a singularity at $P_{1}=P_{1}^{*}$ given by (2.32), we propose a new expansion of the solutions in the vicinity of this point [12]. The asymptotic behavior of the outer solution (2.15) when $\left|P_{1}-P_{1}^{*}\right| \rightarrow$ 0 indicates that the singularity appears when $P_{1}-P_{1}^{*}=O\left(\varepsilon^{1 / 2}\right)$ and suggests that the appropriate scalings in this critical regime are:

$$
\begin{aligned}
& \delta_{1}=\varepsilon\left(\delta_{1}^{*}+\varepsilon^{1 / 2} \Gamma_{1}+\varepsilon \Gamma_{2}+\cdots\right), \\
& x\left(s, \varepsilon^{1 / 4}\right)=\varepsilon^{3 / 4}\left(X_{1}(s)+\varepsilon^{1 / 4} X_{2}(s)+\varepsilon^{1 / 2} X_{3}(s)+\cdots\right),
\end{aligned}
$$

where $\delta_{1}=\delta_{1}^{*}\left(\sigma_{1}, \omega_{1}\right)$ is defined by the condition $P_{1}\left(\delta_{1}^{*}, \sigma_{1}, \omega_{1}\right)=P_{1}^{*}$. Introducing (2.16), (2.17), (2.50), (2.51) into equations (2.10) and equating like powers of $\varepsilon^{1 / 4}$, we find that

$$
x=\varepsilon^{3 / 4}\left[\left(\phi_{1}+O\left(\varepsilon^{1 / 2}\right)\right) u_{1}^{0}+\left(\phi_{3}+O\left(\varepsilon^{1 / 2}\right)\right) u_{3}^{0}\right]+\varepsilon\left(\phi_{2}+O\left(\varepsilon^{1 / 2}\right)\right) u_{2}^{0}+O\left(\varepsilon^{5 / 4}\right),
$$

where the amplitudes $\phi_{1}, \phi_{2}, \phi_{3}$ are obtained from the solvability conditions:

$$
\phi_{2}=\alpha_{2}^{*}
$$


and $\alpha_{2}^{*}$ is defined by (2.31), $\phi_{3}$ is simply related to $\phi_{1}$ by

$$
\phi_{3}=-\frac{B_{2} \phi_{1}}{B_{1}}
$$

and $\phi_{1}$ satisfy an equation of the form,

$$
\phi_{1}^{4} S_{2}+\phi_{1}^{2} S_{1} R+S_{0}=0
$$

$S_{2}, S_{1}, S_{0}$ represent complex expressions of the coefficients appearing in the successive solvability conditions. $R=R\left(\Gamma_{1}\right)$ is related to $\lambda$ by,

$$
\lambda-\lambda_{1}=\varepsilon P_{1}^{*}+\varepsilon^{3 / 2} R+O\left(\varepsilon^{2}\right) .
$$

When $S_{0} S_{2}<0$, there exists a unique positive solution for $\phi_{1}^{2}$ which is defined for $R \gtrless 0$. However when $S_{2} S_{0}>0$, there exist two positive solutions for $\phi_{1}^{2}$ which are defined for $R>0$ if $S_{0} S_{1}<0$ or for $R<0$ if $S_{0} S_{1}>0$. These two branches of solutions admit a limit point given by, $R^{2}=4 S_{0} S_{2} / S_{1}^{2}$. Moreover, when $|R| \rightarrow \infty$, the inner solutions admit the two following limits:

$$
\begin{aligned}
& \phi_{1}^{2}=O\left(|R|^{-1}\right), \\
& \phi_{1}^{2}=O(|R|) .
\end{aligned}
$$

We have verified that $(2.57 \mathrm{~b})$ approaches the inner limit of the outer solution which is given by (2.30). Composite expansion can be formed from the inner and outer expansions, as it is proposed by the method of matched asymptotic expansions [12]. The second limit $(2.57 \mathrm{~b})$ connects large amplitude solutions which are not described by our analysis. Figure 3 represents the complete bifurcation diagram of the steady state solutions.

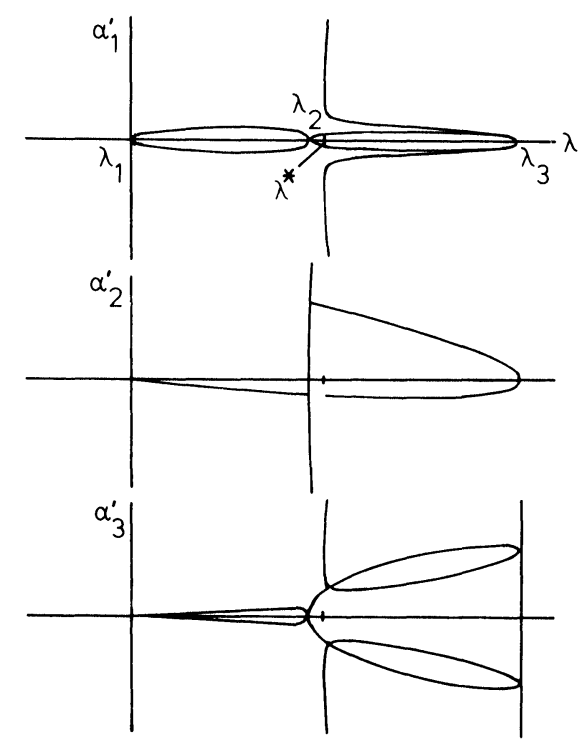

FIG. 3. Composite bifurcation diagram. Taking into account the inner description of the steady state solutions near the singular point defined by (2.32)-(2.33), a uniform representation of the solutions can be obtained. $x \simeq \sum_{j=1}^{3} \alpha_{j}^{\prime} u_{j}^{0}$, where $\alpha_{j}^{\prime} \simeq \varepsilon \alpha_{j}(j=1,2,3)$ for all values of $\lambda$ except near the singular point $\lambda=\lambda^{*}+O\left(\varepsilon^{3 / 2}\right): \alpha_{1}^{\prime} \simeq \varepsilon^{3 / 4} \beta_{1}, \alpha_{3}^{\prime} \simeq \varepsilon^{3 / 4} \beta_{3}, \alpha_{2}^{\prime} \simeq \varepsilon \alpha_{2}^{*}$ and near the primary bifurcation points $\lambda=\lambda_{2}+O\left(\varepsilon^{2}\right):$ $\alpha_{1}^{\prime} \simeq \varepsilon \beta_{1}, \alpha_{3}^{\prime} \simeq \varepsilon \beta_{3}, \alpha_{2}^{\prime} \simeq \varepsilon^{3 / 2} \beta_{2}$ and $\lambda=\lambda_{3}+O\left(\varepsilon^{2}\right): \alpha_{1}^{\prime} \simeq \varepsilon \beta_{1}, \alpha_{2}^{\prime}=\varepsilon \beta_{2}, \alpha_{3}^{\prime} \simeq \varepsilon^{3 / 2} \beta_{3}$. 
3. A model system presenting a triple eigenvalue. In this section, we give an example of a reaction-diffusion model presenting a triple eigenvalue.

Consider the following four variable system

$$
\begin{aligned}
& \frac{\partial}{\partial t} X_{1}=F\left(X_{1}, Y_{1}, \lambda\right)+D_{1} \frac{\partial^{2}}{\partial r} X_{1}+k_{1}\left(X_{2}-X_{1}\right), \\
& \frac{\partial}{\partial t} Y_{1}=G\left(X_{1}, Y_{1}, \lambda\right)+D_{2} \frac{\partial^{2}}{\partial r} Y_{1}+k_{2}\left(Y_{2}-Y_{1}\right), \\
& \frac{\partial}{\partial t} X_{2}=F\left(X_{2}, Y_{2}, \lambda\right)+D_{1} \frac{\partial^{2}}{\partial r} X_{2}+k_{1}\left(X_{1}-X_{2}\right), \\
& \frac{\partial}{\partial t} Y_{2}=G\left(X_{2}, Y_{2}, \lambda\right)+D_{2} \frac{\partial^{2}}{\partial r^{2}} Y_{2}+k_{2}\left(Y_{1}-Y_{2}\right), \quad 0 \leqq r \leqq 1, \\
& \frac{\partial}{\partial r} X_{1}=\frac{\partial}{\partial r} X_{2}=\frac{\partial}{\partial r} Y_{1}=\frac{\partial}{\partial r} Y_{2}=0 \quad \text { at } r=0,1,
\end{aligned}
$$

where $F(X, Y, \lambda)$ and $G(X, Y, \lambda)$ correspond to the kinetic equations of the so called "Brussellator" model [14]:

$$
\begin{aligned}
& F(X, Y, \lambda) \equiv F(X, Y, B)=A+X^{2} Y-(B+1) X, \\
& G(X, Y, \lambda) \equiv G(X, Y, B)=B X-X^{2} Y .
\end{aligned}
$$

A uniform steady state solution of (3.1) is

$$
X_{1}=X_{2}=A ; \quad Y_{1}=Y_{2}=\frac{B}{A} .
$$

Its stability can be determined by linear stability analysis. Setting

$$
X_{j}=A+u_{j}(r) e^{\sigma t}, \quad Y_{j}=B / A+v_{j}(r) e^{\sigma t}, \quad j=1,2
$$

in (3.1), and neglecting the nonlinear terms in $u_{j}(r), v_{j}(r)$, we find that $u_{j}(r), v_{j}(r)$ $(j=1,2)$ must satisfy the linear eigenvalue problem

$$
\begin{gathered}
\left(D \frac{d^{2}}{d r^{2}}+L+K-\sigma I\right)\left(\begin{array}{l}
u_{1} \\
v_{1} \\
u_{2} \\
v_{2}
\end{array}\right)=0, \quad 0 \leqq r \leqq 1, \\
\frac{d}{d r} u_{j}=\frac{d}{d r} v_{j}=0 \quad(j=1,2) \quad r=0,1
\end{gathered}
$$

where

$$
\begin{gathered}
L \equiv\left(\begin{array}{ccccc}
B-1 & A^{2} & 0 & 0 \\
-B & -A^{2} & 0 & 0 \\
0 & 0 & B-1 & A^{2} \\
0 & 0 & -B & -A^{2}
\end{array}\right), \quad K \equiv\left(\begin{array}{rrrrr}
-k_{1} & 0 & k_{1} & 0 \\
0 & -k_{2} & 0 & k_{2} \\
k_{1} & 0 & -k_{1} & 0 \\
0 & k_{2} & 0 & -k_{2}
\end{array}\right) .
\end{gathered}
$$


The eigenfunctions of (3.4) are

$$
\left(\begin{array}{l}
u_{j} \\
v_{j}
\end{array}\right)=\left(\begin{array}{c}
1 \\
c_{n j}
\end{array}\right) \cos n \pi r, \quad j=1,2, \quad n=0,1,2 \cdots,
$$

provided the eigenvalues $\sigma=\sigma_{n}$ satisfy

$$
\operatorname{det}\left(-D n^{2} \pi^{2}-\sigma_{n} I+L+K\right)=0 .
$$

The trivial solution (3.2) is stable if $\operatorname{Re}\left(\sigma_{n}\right)<0$ for all $n$ but is unstable if not.

The eigenvalues $\sigma_{n}$ satisfy the following characteristic equation:

$$
f_{1}\left(\sigma_{n}\right) \cdot f_{2}\left(\sigma_{n}\right)=0
$$

where

$$
\begin{gathered}
f_{1}\left(\sigma_{n}\right)=\sigma_{n}^{2}-\sigma_{n}\left\{B-1-A^{2}-\left(D_{1}+D_{2}\right) n^{2} \pi^{2}\right\} \\
+\left(B-1-D_{1} n^{2} \pi^{2}\right)\left(-A^{2}-D_{2} n^{2} \pi^{2}\right)+A^{2} B \\
f_{2}\left(\sigma_{n}\right)=\sigma_{n}^{2}-\sigma_{n}\left\{B-1-A^{2}-\left(D_{1}+D_{2}\right) n^{2} \pi^{2}-2\left(k_{1}+k_{2}\right)\right\} \\
+\left(B-1-2 k_{1}-D_{1} n^{2} \pi^{2}\right)\left(-A^{2}-2 k_{2}-D_{2} n^{2} \pi^{2}\right)+A^{2} B
\end{gathered}
$$

Using $B$ as a bifurcation parameter, the results are as follows:

1. One real eigenvalue becomes positive when

$$
B>B_{n}=1+\frac{A^{2} D_{1}}{D_{2}}+\frac{A^{2}}{D_{2} n^{2} \pi^{2}}+D_{1} n^{2} \pi^{2}
$$

or

$$
B>B_{n}^{\prime}=\left(1+2 k_{1}+D_{1} n^{2} \pi^{2}\right)\left(1+\frac{A^{2}}{\left(2 k_{2}+D_{2} n^{2} \pi^{2}\right)}\right) .
$$

2. A complex eigenvalue has a positive real part when

$$
B>\tilde{B}_{n}=1+A^{2}+\left(D_{1}+D_{2}\right) n^{2} \pi^{2}, \quad \tilde{B}_{n}<B_{n}
$$

or

$$
B>\tilde{B}_{n}^{\prime}=1+A^{2}+2\left(k_{1}+k_{2}\right)+\left(D_{1}+D_{2}\right) n^{2} \pi^{2}, \quad \tilde{B}_{n}^{\prime}<B_{n}^{\prime} .
$$

A zero eigenvalue of multiplicity 3 appears, for example, when

$$
B^{*}=B_{1}^{\prime}=B_{2}=B_{3} \text {. }
$$

This is possible if $A$ and $k_{2}$ are chosen such that

$$
\begin{gathered}
A=9 \pi^{2}\left(D_{1} D_{2}\right)^{1 / 2}, \\
k_{2}=\frac{1}{2}\left\{D_{2} \pi^{2}+\frac{\left(1+2 k_{1}+D_{1} \pi^{2}\right) D_{1} D_{2} 36 \pi^{4}}{12 D_{1} \pi^{2}\left(1+3 D_{1} \pi^{2}\right)-2 k_{1}}\right\}, \\
12 D_{1} \pi^{2}\left(1+3 D_{1} \pi^{2}\right)-2 k_{1}>0 .
\end{gathered}
$$

Under these conditions

$$
B^{*}=1+D_{1} 4 \pi^{2}+D_{1} 9 \pi^{2}+D_{1}^{2} 36 \pi^{4} .
$$

$B^{*}$ corresponds to the first bifurcation point if

$$
B^{*}<\left\{B_{j}^{\prime}, B_{l}, \tilde{B}_{0}\right\}, \quad j=1,2,3, \cdots, \quad l=1,2 \cdots .
$$


For $D_{1}=0.04, D_{2}=0.5, k_{1}=1$, we find

$$
A \simeq 8.37, \quad k_{2} \simeq 11.79, \quad B^{*}=B_{1}^{\prime}=B_{2}=B_{3} \simeq 11.74 \text {. }
$$

$B^{*}$ corresponds to the first bifurcation point.

4. Discussion. Our first interest is to study from the bifurcation theory point of view the possible transition between nonsymmetric and symmetric chemical gradients when the bifurcation parameter gradually changes from a stable to an unstable reference state. Recent works [2] have showed that such a transition is possible, only one symmetric solution can be expected to be stable-and therefore observed experimentally [3] - and this transition is still possible even if a parameter is nonuniformly distributed in the system. The principal purpose of this paper was to investigate the transformation from nonsymmetric to symmetric steady state solutions when three primary branches of steady states, characterized by an increasing wave number, are interacting i.e. near a triple degenerate bifurcation point. The analysis showed that:

(i) a primary branch of nonsymmetric solutions emerging at $\lambda=\lambda_{1}$ may connect the primary branch of symmetric solutions, appearing at $\lambda=\lambda_{2}$ if the singular point $\lambda=\lambda^{*}=\lambda_{1}+\varepsilon P_{1}^{*}+O\left(\varepsilon^{2}\right)>\lambda_{2}$ where $P_{1}^{*}$ is given by (2.32) (see Fig. 3).

(ii) By contrast to triple degenerate bifurcation problems in two or three space dimensions [4], [15], we find no tertiary branches of steady state solutions.

Further analysis is however required in order to complete our preliminary results. First, the stability of the various solutions can be studied by considering the linearized equations of evolution. Second, it could be interesting to compare the informations of our analysis of bifurcation near a triple eigenvalue to those which can be obtained near double eigenvalues [2], [5], [8], [9], [16] or by numerical simulations of simple two-variable models [3], [5], [10]. Indeed, quite different behaviors can be observed; for example, when $\eta=\left|\lambda_{3}-\lambda_{1}\right|, \lambda-\lambda_{1}=O(\eta), \eta \ll 1$ but $\lambda_{2}-\lambda_{1}=O(1)$, the primary branch of solutions, emerging at $\lambda=\lambda_{3}$, presents a bifurcation to secondary solutions which are defined on both sides of the bifurcation point [5]. This behavior is not observed when $\lambda_{2}-\lambda_{1}=O(\eta)$ i.e. in the vicinity of a triple degenerate bifurcation point since the secondary states are only defined on one side of the bifurcation point. In future work, we intend to explore further these qualitative modifications of the bifurcation diagram.

Appendix A: Definitions of $P_{j}(j=1,2,3), A_{1}, A_{2}, B_{1}, B_{2}$, and $C_{1}$. The coefficients $P_{j}(j=1,2,3), A_{1}, A_{2}, B_{1}, B_{2}, C_{1}$, appearing in the bifurcation equations (2.26), are defined by

$$
\begin{aligned}
& \left.\left(\delta_{1} M_{1}+\sigma_{1} M_{2}+\omega_{1} M_{3}\right) p_{j}, p_{j}^{*}\right)=P_{j}\left(M_{1} p_{j}, p_{j}^{*}\right), \\
& A_{1}=\frac{1}{2}\left(\left\{Q_{2}\left(p_{1}, p_{2}, 0,0,0\right)+Q_{2}\left(p_{2}, p_{1}, 0,0,0\right)\right\}, p_{1}^{*}\right) /\left(M_{1} p_{1}, p_{1}^{*}\right), \\
& A_{2}=\frac{1}{2}\left(\left\{Q_{2}\left(p_{3}, p_{2}, 0,0,0\right)+Q_{2}\left(p_{2}, p_{3}, 0,0,0\right)\right\}, p_{1}^{*}\right) /\left(M_{1} p_{1}, p_{1}^{*}\right), \\
& B_{1}=\frac{1}{2}\left(Q_{2}\left(p_{1}, p_{1}, 0,0,0\right), p_{2}^{*}\right) /\left(M_{1} p_{2}, p_{2}^{*}\right), \\
& B_{2}=\frac{1}{2}\left(\left\{Q_{2}\left(p_{1}, p_{3}, 0,0,0\right)+Q_{2}\left(p_{3}, p_{1}, 0,0,0\right)\right\}, p_{2}^{*}\right) /\left(M_{1} p_{2}, p_{2}^{*}\right), \\
& C_{1}=\frac{1}{2}\left(\left\{Q_{2}\left(p_{1}, p_{2}, 0,0,0\right)+Q_{2}\left(p_{2}, p_{1}, 0,0,0\right)\right\}, p_{3}^{*}\right) /\left(M_{1} p_{3}, p_{3}^{*}\right) .
\end{aligned}
$$

$M_{j}(j=1,2,3)$ and $Q_{2}(x, x, 0,0,0)$ have been defined by $(2.18)-(2.19) . p_{j}(j=1,2,3)$ correspond to constant vectors defined by $(2.7)$ and $p_{j}^{*}(j=1,2,3)$ are constant vectors associated with the three solutions of the adjoint equation:

$$
L_{0}^{*} u_{n}^{*}=0 \text {. }
$$


The solutions of (A.2) are of the form

$$
u_{n}^{*}=c_{n} p_{n}^{*} \cos n \pi s, \quad n=1,2, \cdots,
$$

where $p_{n}^{*}$ is a constant vector satisfying:

$$
\left\{\left(\frac{\partial F}{\partial X_{j}}\right)_{X_{0}, \lambda^{*}}^{*}-\left(D / l^{2}\right) n^{2} \pi^{2}\right\} p_{n}^{*}=0
$$

$\left(\partial F / \partial X_{j}\right)_{X_{0}, \lambda}^{*}$ denotes the adjoint operator to $\left(\partial F / \partial X_{j}\right)_{X_{0}, \lambda} * c_{n}$ is a constant coefficient defined by

$$
c_{n}=\frac{2}{\left(p_{n}, p_{n}^{*}\right)}
$$

Appendix B: Definition of $\boldsymbol{B}_{3}$. The constant vectors $p_{0}$ and $p_{4}$ are obtained by solving

$$
\begin{gathered}
\left(\frac{\partial F}{\partial X_{j}}\right)_{X_{0}, \lambda^{*}} p_{0}=-Q_{2}\left(p_{2}, p_{2}, 0,0,0\right) \frac{1}{2}, \\
\left(\left(\frac{\partial F}{\partial X_{j}}\right)_{X_{0}, \lambda^{*}}-16 \frac{D \pi^{2}}{l^{2}}\right) p_{4}=-Q_{2}\left(p_{2}, p_{2}, 0,0,0\right) \frac{1}{2},
\end{gathered}
$$

and the new coefficient $B_{3}$ appearing in the bifurcation equation (2.40) is given by

$$
\begin{aligned}
B_{3}=\left(\left\{Q_{2}\left(p_{0}, p_{2}, 0,0,0\right)+Q_{2}\left(p_{2}, p_{0}, 0,0,0\right)\right.\right. \\
+\left[Q_{2}\left(p_{4}, p_{2}, 0,0,0\right)+Q_{2}\left(p_{2}, p_{4}, 0,0,0\right)\right]^{\frac{1}{2}} \\
\left.\left.\quad+Q_{3}\left(p_{2}, p_{2}, p_{2}, 0,0,0\right) \frac{3}{4}\right\}, p_{2}^{*}\right) /\left(M_{1} p_{2}, p_{2}^{*}\right) .
\end{aligned}
$$

Appendix C: Definition of $\boldsymbol{C}_{2}$. The coefficient $C_{2}$ appears in the bifurcation equations (2.46) and is given by:

$$
\begin{aligned}
C_{2}=( & \left\{Q_{2}\left(q_{0}, p_{3}, 0,0,0\right)+Q_{2}\left(p_{3}, q_{0}, 0,0,0\right)\right. \\
+ & {\left[Q_{2}\left(p_{6}, p_{3}, 0,0,0\right)+Q_{2}\left(p_{3}, p_{6}, 0,0,0\right)\right]^{\frac{1}{2}} } \\
& \left.\left.+Q_{3}\left(p_{3}, p_{3}, p_{3}, 0,0,0\right) \frac{3}{4}\right\}, p_{3}^{*}\right) /\left(M_{1} p_{3}, p_{3}^{*}\right),
\end{aligned}
$$

where $q_{0}$ and $p_{6}$ are two new constant vectors obtained by solving the following equations:

$$
\begin{aligned}
& \left(\frac{\partial F}{\partial X_{j}}\right)_{X_{0}, \lambda^{*}} q_{0}=-Q_{2}\left(p_{3}, p_{3}, 0,0,0\right) \frac{1}{2}, \\
& \left(\left(\frac{\partial F}{\partial X_{j}}\right)_{X_{0}, \lambda^{*}}-36 \frac{D \pi^{2}}{l^{2}}\right) p_{6}=-Q_{2}\left(p_{3}, p_{3}, 0,0,0\right) \frac{1}{2} .
\end{aligned}
$$

Acknowledgments. T.E. is Chargé de Recherches du Fonds National de la Recherche Scientifique (Belgium). We thank Professor E. L. Reiss for the critical reading of the manuscript.

\section{REFERENCES}

[1] L. BAuer, H. B. Keller AND E. L. Reiss, Multiple eigenvalues lead to secondary bifurcation, SIAM Rev., 17 (1975), pp. 101-122.

[2] T. ERNEUX AND D. S. COHEN, Imperfect bifurcation near a double eigenvalue: transition between nonsymmetric and symmetric patterns, this Journal, this issue, pp. 1042-1060. 
[3] T. ERneux And J. HiernauX, Transition from polar to duplicate patterns, J. Math. Biol., 9 (1980), pp. 193-211.

[4] T. ERNEUX AND E. L. REISS, Splitting of steady multiple eigenvalues may lead to periodic cascading bifurcation, this Journal, 43 (1983), pp. 613-624.

[5] H. Fuji, M. Mimura AND Y. Nishiura, A picture of the global bifurcation diagram in ecological interacting and diffusing systems, Physica D, 5 (1982), pp. 1-42.

[6] A. GIERER, Biological features and physical concepts of pattern formation exemplified by Hydra, Curr. Top. Devel. Biol., 11 (1977), pp. 17-59.

[7] - Generation of biological patterns and form: Some physical, mathematical and logical aspects, Prog. in Biophys. and Mol. Biology, 37 (1981), pp. 1-47.

[8] R. A. Goldstein, M. A. Huerta And J. C. NeAring, Secondary bifurcation near multiple eigenvalues, 1983, to appear.

[9] J. P. KEENER, Secondary bifurcations in nonlinear diffusion reaction equations, Stud. Appl. Math., 55 (1976), pp. 187-211.

[10] M. Kubicek, V. RYZler AND M. MAREK, Spatial structures in a reaction-diffusion system. Detailed analysis of the "Brussellator", Biophys. Chem., 8 (1978), pp. 235-246.

[11] T. J. MAHAR AND B. J. MATKOWSKY, A model biochemical reaction exhibiting secondary bifurcation, this Journal, 32 (1977), pp. 394-404.

[12] B. J. MATKOWSKY AND E. L. REISs, Singular perturbations of bifurcations, this Journal, 33 (1977), pp. 230-255.

[13] H. MEINHARDT, Models for the ontogenetic development of higher organisms, Rev. Physiol. Biochem. Pharmacol., 80 (1978), pp. 48-104.

[14] G. NiCOlis AND I. Prigogine, Self-Organization in Non-Equilibrium Systems, John Wiley, New York, 1977.

[15] E. L. ReISs, Cascading bifurcations, this Journal, 43 (1983), pp. 57-65.

[16] D. G. SChAEFFER AND M. A. Golubitsky, Bifurcation analysis near a double eigenvalue of a model chemical reaction, Arch. Rat. Mech. Anal., 75 (1981), pp. 315-348.

[17] L. WOLPERT, Positional information and the spatial pattern of cellular differentiation, J. Theoret. Biol., 25 (1969), pp. 1-47.

[18] — Pattern formation in biological development, Scientific American, October, 1978, pp. 124-137. 